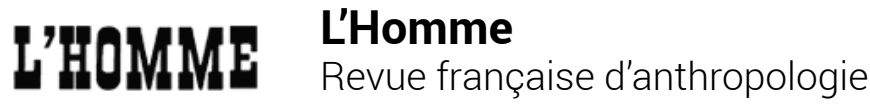

160 | octobre-décembre 2001

Droit, coutume, mémoire

\section{Didier Fassin, Les Enjeux politiques de la santé. Études} sénégalaises, équatoriennes et françaises

Paris, Karthala, 2000, 344 p., bibl., réf. (« Hommes et Sociétés »)

\section{Christine Arnault}

\section{OpenEdition}

\section{Journals}

Édition électronique

URL : http://journals.openedition.org/lhomme/7751

DOI : 10.4000/lhomme.7751

ISSN : 1953-8103

Éditeur

Éditions de l'EHESS

Édition imprimée

Date de publication : 1 janvier 2001

Pagination : 215-216

ISBN : 2-7132-1391-6

ISSN : 0439-4216

Référence électronique

Christine Arnault, «Didier Fassin, Les Enjeux politiques de la santé. Études sénégalaises, équatoriennes et françaises », L'Homme [En ligne], 160 | octobre-décembre 2001, mis en ligne le 31 mai 2007, consulté le 24 septembre 2020. URL : http://journals.openedition.org//homme/7751 ; DOI : https://doi.org/ 10.4000/lhomme.7751

Ce document a été généré automatiquement le 24 septembre 2020

(c) École des hautes études en sciences sociales 


\title{
Didier Fassin, Les Enjeux politiques de la santé. Études sénégalaises, équatoriennes et françaises
}

\author{
Paris, Karthala, 2000, 344 p., bibl., réf. (« Hommes et Sociétés »)
}

\section{Christine Arnault}

DANS cet ouvrage, Didier Fassin se propose de montrer que la santé ou un problème d'ordre sanitaire peut remettre en cause les institutions politiques, et ce dans trois pays : la France, le Sénégal et l'Équateur. Il fait voir comment le politique est mis à l'épreuve à travers des questions de santé, bien que cette mise à l'épreuve ne soit pas visible au premier abord.

2 L'auteur reprend à son compte la thèse foucaldienne selon laquelle le biologique est étroitement lié au pouvoir, l'ordre social s'inscrit dans l'être physique et le politique intervient dans le champ de la santé. La santé est soumise à des facteurs politicogéographico-culturels de telle sorte que selon que l'on est né dans un pays pauvre ou dans un pays riche, que l'on vit dans un pays du Nord ou du Sud, que l'on appartient à une classe sociale favorisée ou défavorisée, enfin selon que l'on est du sexe féminin ou du sexe masculin, l'état de santé peut varier considérablement. Toutefois, la comparaison est rendue difficile du fait que la santé ne se définit pas partout de la même manière : les statistiques sanitaires ne sont pas homogènes et les priorités que les États se fixent pour répondre aux problèmes de prévention ou de soins ne le sont pas non plus.

Sont ainsi examinées quatre « épreuves politiques de la santé ». La première est celle de l'inégalité ou de l'incorporation de l'ordre politique, autrement dit, de la transcription dans les corps des disparités sociales. La seconde, celle du pouvoir, met en scène les détenteurs de savoirs thérapeutiques; à cette occasion sont abordées les différentes configurations de l'espace thérapeutique. La troisième, celle du gouvernement, engage la gestion collective de la santé; la réflexion porte sur la délimitation du champ sanitaire et sur des réalités non prises en compte - comme les processus d'exclusion et d'inclusion produits par ces politiques -, alors qu'elles concernent le rapport entre le 
corps et le social. Enfin, la quatrième épreuve est celle de la cité : l'auteur pose la question de la participation des individus sous la pression des pouvoirs publics locaux ou internationaux qui, au nom d'une idéologie populiste ou d'une utopie communautaire, imposent un modèle de citoyenneté. L'épidémie de sida est un cas paradigmatique des questions posées aux politiques de santé dans la mesure où il leur fait parcourir ces quatre épreuves.

Didier Fassin suggère trois niveaux de lecture de ces épreuves de la santé : celui de la production politique de la santé, et plus précisément le façonnement de la santé individuelle et collective par la société ; celui de la production des politiques de santé, c'est-à-dire les réponses données par celles-ci en fonction de ce que la société considère comme problèmes de santé ; enfin, celui de la construction politique de la santé. Audelà des trois pays étudiés, l'auteur examine une variété très riche de thèmes et de terrains.

5 Comme il le souligne, il ne s'agit pas de procéder à une quelconque comparaison entre les pays, mais à un aller et retour entre les cultures afin de comprendre ce qui conduit les individus a ne pas se soigner correctement; il montre par exemple la difficulté, voire l'impossibilité, de recourir aux structures sanitaires lorsqu'on se trouve dans une situation de précarité économique. Cette difficulté ne concerne pas seulement les pays pauvres ou les pays du tiers monde, mais touche également les pays occidentaux. Didier Fassin se livre à ce sujet à une critique très sévère des méthodes employées dans tel ou tel pays subsaharien par certaines organisations internationales, ou par les États africains eux-mêmes, pour tenter d'enrayer l'épidémie de sida. De même, il refuse que soit pris en compte de façon isolée le facteur culturel pour expliquer les réticences des populations face à la médecine officielle ou face aux services sanitaires. Pour lui, il convient d'être prudent et de ne pas établir de lien entre immigration et sida, ou Afrique subsaharienne et sida.

\section{AUTEUR}

\section{CHRISTINE ARNAULT}

Paris. 